\title{
Creating eLearning material with optimal utilisation of limited resources
}

\section{Gunawardena}

i-Context Content Convergance, PVT., Sri Lanka.

E-mail address: cgunawardena@i-context.net

eHealth Sri Lanka 2010,1(suppl.1):S13

DOI: http://dx.doi.org/10.4038/sljbmi.v1i0.3547

Only the Abstract is available

\begin{abstract}
Sri Lanka is a country with comparatively high standards of healthcare, but a poor distribution of resources. While gaining access to expert trainers is relatively easy in the western province, the same cannot be said of regions like the eastern and north central provinces. With access to broadband crossing all regional and geographic boundaries and an $84 \%$ penetration rate for mobile phones, internet access is becoming the one resource that is uniformly distributed across the island. An eLearning solution therefore is the only practical means to ensure that training resources are available to everyone, anytime, anywhere in the island. The paper proposes a hosted eLearning solution incorporating video based lessons supplemented by text, animations and voiceovers to provide postgraduate level training for medical personnel. The process of creating training material will be non intrusive and not require any additional time or expensive resources as it will be primarily based on capturing of actual cases including consultations, surgery and other day to day activities as videos. A simple webcam attached to a laptop or even a relatively high resolution mobile phone with sufficient storage capacity is all that is required to capture the core training content. Assembly of the training course using these videos can be done by eLearning domain specialists, along with instructional design and preparation of rich content such as animations; only the knowledge and information needs to be provided by subject matter experts. The proposed approach ensures that the most valuable resource consumed in this process, the time of the expert practitioner, is used optimally and in a manner that has the least impact on his or her primary responsibility, that of providing care to patients.
\end{abstract}

Keywords - resource, actual cases, access to broadband 